\title{
An E-Conversation with Prof. Farhang Shadman, Regents Professor, University of Arizona, Tucson, AZ
}

\author{
Subhas K. Sikdar
}

Published online: 4 February 2014

(C) Springer-Verlag Berlin Heidelberg (outside the USA) 2014

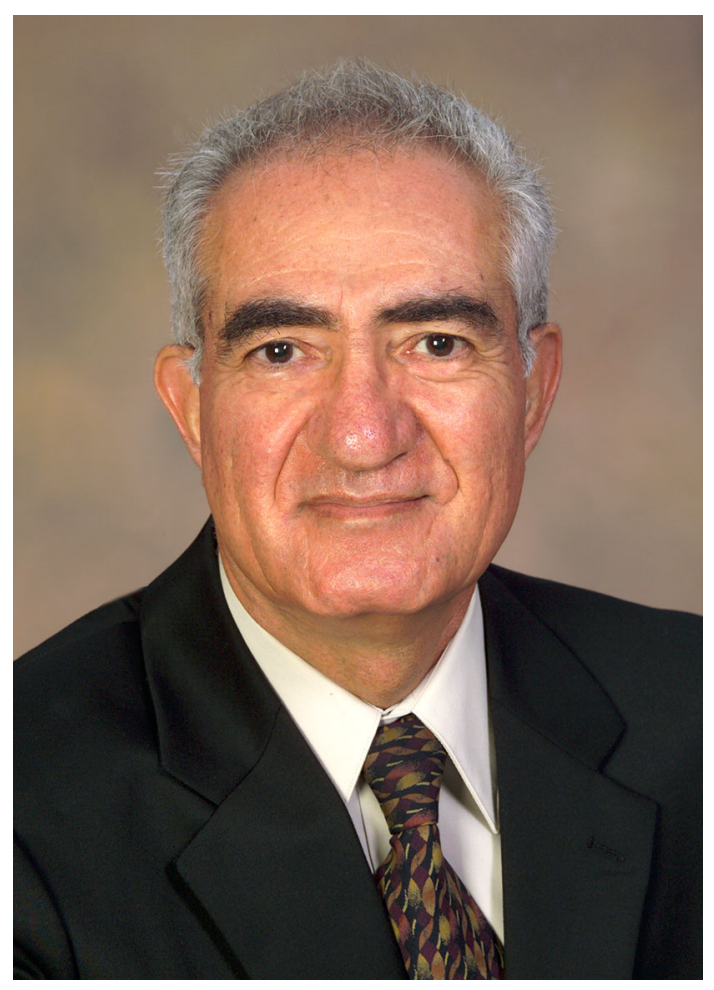

Dr. Shadman is a Regents Professor of Chemical and Environmental Engineering (joint appointment as a Professor of Optical Sciences) at the University of Arizona and the Director of the Engineering Research Center for Environmentally Benign Semiconductor Manufacturing. He was also the founder and Director of Sematech Center for Defect Control in Semiconductor Manufacturing from 1986 to 1996.

S. K. Sikdar ( ()

University of Arizona, Tucson, AZ, USA

e-mail: sikdar.subhas@epa.gov
SKS: Farhang, as a well-known researcher and a Center Director in charge of sustainable manufacturing methods in the semiconductor sector, you have worked closely with many researchers and executives in the high technology industries. You are very familiar with the fact that most of the large corporations in this sector are paying a great deal of attention to sustainability in their operations. Can you tell us if there is a common view of sustainability among companies across this sector, and how your own view coincides or differs from theirs?

FS: Semiconductor industry, the key manufacturing arm of the information technology, has a number of unique and unprecedented characteristics. First of all, its impressive rate of growth is much greater than that of any industry in the past. Secondly the time from concept/idea to highvolume manufacturing and introduction of product to market is relatively short (typically less than 8 years and continuously decreasing with every new generation of products). Finally, the number of feed chemicals used in the manufacturing of integrated circuit (IC) chips is truly impressive and unprecedented; the usage of natural resources for the advanced IC fabrication is also very large compared to most conventional industries; this last characteristic is closely related to the environmental impact potentials that this industry has been facing and to a great extent has been successfully resolved.

In the face of these challenges, the industry has taken an approach which is also unique and unprecedented. The industry, even at its highest level of management, has acknowledged that some of these environmental issues could potentially become critical in manufacturing sustainability. It is impressive that sustainability in this is not defined as a strategy to maintain the status quo; but rather the ability to sustain the desired rate of growth. The industry has recognized that the sustainability challenges 
are not the type that can be solved by each company independently. The first reason is that the suppliers (both chemical and equipment companies), who play the key role in implementing the new technology into manufacturing methods, are common and shared by IC fabrication companies. The second and more pressing issue is that the money and human resources needed to solve the complex technical issues of sustainability are too large for individual companies to afford.

The consequence has been a visionary and unique approach to the development of a set of common national and international documents that define the key sustainability issues and set specific goals and time tables to find solutions and bring them into the high-volume manufacturing stage. These documents (called technology roadmaps) are continuously being updated by experts from both industry and research community in the national and international levels. These roadmap documents are valuable guides to researchers for their research planning and to suppliers companies for the design of new tools and techniques. I believe that this organized and visionary approach, which is a good example of proactive approach to sustainability, could serve as a model for other industries, particularly in the high-technology sector, to follow.

SKS: In the nineties when pollution prevention was how we used to describe our objectives to design cleaner manufacturing, many corporation popularized the adage that pollution prevention pays, i.e. by practicing pollution prevention, we not only protect the environment, we save money in the process. Now, extending this idea to sustainability, how in your view does economics play a decision making part?

FS: In our view, sustainability has three major components: The first one is the competitiveness in performance; no industry or manufacturing can remain sustainable if its product cannot compete in quality and functionality in the marketplace. The second one is the environmental and social footprint; this includes the availability of the required resources and the environmental and social impact of its manufacturing and its products. The third component is the cost and economic bottom line. While these three factors may not be totally orthogonal and independent, they represent the distinct and measurable metrics of sustainability. In my opinion, in the long run, all three factors have equal weight and should be given equal emphasis and importance. Cost is clearly the most easily measurable, quantifiable, and therefore reportable metric; however, it is not the only factor or even the dominant factor in sustainability. Instead, these three factors are three legs on which a stable and sustainable industry can stand.

SKS: Very well. I want to focus on the environmental and social footprints of the products and the manufacturing sites. Environmental footprint is not hard to quantify. This is mostly negative impacts, and we add up all the effects of emissions and discharges and somehow express it, either in health terms or its related monetized term. However, it would not be hyperbole to say that electronic industry has made life in general more productive, comfortable, and fun, in addition to creating employment and opportunities that did not exist before. This is a social benefit. Perhaps it is difficult to quantify it but when one considers societal impact, the negative should be compared to the positive. What is your opinion of how industry and researchers handle or should handle this issue.

FS: Quantitative evaluation of the sustainability of an industry is very challenging and in some cases not possible both from environmental impact as well as from social points of view. Even on the environmental aspect that you have mentioned, the footprint of a particular industry or manufacturing depends on where you draw what we call "the environmental assessment boundary". If you draw this boundary around a process or a factory, most manufacturing processes will show net negative environmental impact due to their emissions and use of resources. However, if you expand the boundary, include the community, and assess the impact in a more global and broader scale, the net impact could turn out to be positive. A classic example is the environmental impact of computers and chip manufacturing. If it is assessed within a factory boundary, it will most probably show a net negative impact due to its inevitable emissions and use of resources. However, if it assessed within a more global boundary, it will show many positive aspects due to the reduction in usage of paper and many other materials and resources that have been made possible by electronic data processing, storage, and communication. We may have to accept the fact that not every impact can be quantized to the same level and therefore some aspects need to be simply judged on their qualitative merit as "positive/negative", "acceptable/unacceptable", and "go/no-go" in any sustainability modeling. We should also expect that this designation may change with time, location, and circumstances, as many societal issues do.

SKS: We alluded to the toxic nature of many chemicals used in manufacturing in this industry. I also know that researchers are trying to determine toxicity potential of these chemicals. How is it being done and is the progress satisfactory so far?

FS: The approach and the progress of semiconductor industry in this area have been remarkable and praiseworthy for two reasons: The first reason is that the semiconductor, unlike most other major industries, has traditionally taken a "proactive" approach to the assessment of the safety of chemicals and has a clear roadmap for testing the effects before bringing potentially hazardous materials and questionable processes into high-volume 
manufacturing. The second reason is that this industry has invested heavily in finding ESH-friendly alternatives any time a potentially problematic material is identified, well in advance and ahead of the deadlines and the pressure of compliance with regulations. A good example is the semiconductor industry's voluntary efforts and major success in reducing the usage and emission of global warming gases. There are many reasons for the choice of this proactive approach by the IC industry.

SKS: Yes of course. I wonder if there is a generalized way of finding substitutes. Is the process essentially Edisonian, or there are methodical experimental or algorithmic approaches to identifying these safe substitutes, especially when they are new chemicals.

FS: For application in semiconductor processing, we can find examples of both approaches. Considering the large number of materials that are explored for any new generation of electronic devices, one can always find cases where the search involves some trial and error in the early stages of R/D. However, a systematic approach is gradually replacing this approach for three reasons: The trial and error approach typically has a high degree of uncertainty in outcome and cannot be a part of a strict time table that IC industry employs. The second reason is that there is an increasingly deeper and better understanding of the properties of chemicals and materials available to researchers to make a systematic search for alternatives possible with a higher degree of confidence. The third and perhaps the most exciting reason is the development and availability of "molecular modeling" tools that can be used to design molecules with the desired properties as alterative to chemicals that need to be replaced. The use of these models for molecular design of environmentally-friendly alternatives is an exciting and key technology area for semiconductor industry.

SKS: I want to ask you two questions from your response to the last two questions. I wonder what you mean by "environmentally benign" chemicals. I can see two possibilities here. First, it could mean chemicals that do not pose a health risk to humans or ecosystems when the latter are exposed to them "reasonably low" doses. I used the lawyerly language here because we know that health risk is dose related. At high doses even water can be toxic. So these chemicals which are benign in this way can be handled by humans without taking any protection. The second possibility is chemicals that are toxic alright but they are destroyed before any worker can be exposed to them. Many chemicals that are currently essential to electronics are highly toxics but this safe guard is assured in manufacturing operations. Are you implying both here?

FS: We usually use the term "environmentally benign processes" or "environmentally benign manufacturing" but not "environmentally benign chemicals" for exactly the reason that you have pointed out. It is the overall usage that needs to be assessed in terms of environmental impact. However, even for the overall processes or manufacturing, the term "benign" indicates the ideal goal which can be asymptotically approached but in most cases we can never reach it $100 \%$.

SKS: The second question has to do with molecular modeling. Are there any early successes stories you can share that can educate us about the potential of this technique for manufacturing in general?

FS: They are many examples of success in this case. One is in the development of new chemistries and chemicals for etching silicon, silicon dioxide, new high-dielectric substrates, and metal layers. The other one is development of new generation of photo-acid generators (PAGs), used in photo-lithography and patterning of substrates in SC manufacturing. These are replacement for PFOS which is a hazardous chemical. Generally, the use of molecular modeling for this purpose involves first identifying the role that each part or section of the molecule needs to play to bring about the overall processing function for which the chemical is being designed. Then the selected functional groups are linked together (in a thermodynamically feasible way) to form the molecule or chemical of choice. This approach can be extended to atomic level as well. For example, in the use of molecular modeling for finding new etch chemicals, the focus is on finding atoms that would create interactions that result in bond breaking and removal of the substrate that needs to be etched.

SKS: I want to now ask you a personal question. From your career profile I note that you did your $\mathrm{PhD}$ in chemical engineering at UC Berkeley and later you were engaged in a business in chemical manufacturing in Iran. Can you share with us how you became interested in environmental issues, and particularly how you gravitated to semiconductor industry-focused research?

FS: I cannot think of any time in my research career when I was not involved in environmental technology. My first research program after graduating from UC Berkeley was on the development of air pollution dispersion models for large urban areas (a joint project with the Science Center of Rockwell International). After joining the General Motors Research Laboratories, I was working on various aspects of automotive emission control technologies and in particular the development of a novel abatement method for removal and destruction of particulate emission from diesel-powered internal combustion engines. After joining the University of Arizona in 1979, I initially worked on methods for abatement of coal power plants emissions. In early 1980s there was a major change in my research interest, as I learned about and became interested in the application of chemical engineering In semiconductor fabrication processes. Initially I led a 
SEMATECH-sponsored Research Center on contamination control in fabrication plants (Fabs) and later became a founder and the director of the current Center that focuses on the environmental aspects of semiconductor manufacturing. What attracted me to semiconductor manufacturing was the unique dynamics of this fast-moving industry, as it presents exciting opportunities to develop new technologies and see them implemented.

SKS: You have done some pioneering work for the semiconductor industry, and have received several outstanding awards. Which of these awards you are particularly proud of, and why?

FS: I have been fortunate to be honored with a number of recognitions and awards, including the Akira-Inoue Award which is given by worldwide SEMI association for major contributions to the field of environmental, safety, and health in semiconductor manufacturing. However, the recognition that is of greatest value to me is the industry's endorsement and support given to our Engineering Research Center for Environmentally Benign Semiconductor Manufacturing for over 18 years (very unique and long life for an ERC). While I have the honor of being associated with managing this center, the credit for accomplishments goes to a large group of university members and a unique multi-disciplinary team of faculty and students who form the research force of this Center.

SKS: Farhang, Thank you very much for sharing with us your expertise, accomplishments, and your thoughts on sustainability issues as they pertain to semiconductor industry. 